\title{
Self-Replicating Spots in the Brusselator Model and Extreme Events in the One-Dimensional Case with Delay
}

\author{
Mustapha Tlidi ${ }^{1, *, \dagger}$, Yerali Gandica ${ }^{2, \dagger}$, Giorgio Sonnino ${ }^{1,+}$, Etienne Averlant ${ }^{1,3, \dagger}$ and \\ Krassimir Panajotov $3,4,+$ \\ 1 Faculté des Sciences, Université Libre de Bruxelles, Campus Plaine, C.P. 231, Brussels B-1050, Belgium; \\ Giorgio.Sonnino@ulb.ac.be (G.S.); eaverlan@ulb.ac.be (E.A.) \\ 2 Namur Center for Complex Systems (naXys), University of Namur, Rempart de la Vierge 8, \\ Namur B-5000, Belgium \\ 3 Department of Applied Physics and Photonics (IR-TONA), Vrije Universiteit Brussel, Pleinlaan 2, \\ Brussels B-1050, Belgium; kpanajot@b-phot.org \\ 4 Institute of Solid State Physics, 72 Tzarigradsko Chaussee Blvd., Sofia 1784, Bulgaria \\ * Correspondence: mtlidi@ulb.ac.be; Tel.: +32-2-650-59-05; Fax: +32-2-650-58-24 \\ + These authors contributed equally to this work.
}

Academic Editor: Raúl Alcaraz Martínez

Received: 29 November 2015; Accepted: 18 February 2016; Published: 27 February 2016

\begin{abstract}
We consider the paradigmatic Brusselator model for the study of dissipative structures in far from equilibrium systems. In two dimensions, we show the occurrence of a self-replication phenomenon leading to the fragmentation of a single localized spot into four daughter spots. This instability affects the new spots and leads to splitting behavior until the system reaches a hexagonal stationary pattern. This phenomenon occurs in the absence of delay feedback. In addition, we incorporate a time-delayed feedback loop in the Brusselator model. In one dimension, we show that the delay feedback induces extreme events in a chemical reaction diffusion system. We characterize their formation by computing the probability distribution of the pulse height. The long-tailed statistical distribution, which is often considered as a signature of the presence of rogue waves, appears for sufficiently strong feedback intensity. The generality of our analysis suggests that the feedback-induced instability leading to the spontaneous formation of rogue waves in a controllable way is a universal phenomenon.
\end{abstract}

Keywords: localized structures; spot self-replication; extreme events; rogue waves

\section{Introduction}

In their seminal 1968 paper, Prigogine and Lefever derived a trimolecular model to describe the formation of dissipative structures. These periodic structures appear spontaneously in dissipative environments and are maintained thanks to a permanent exchange of matter and/or energy with their surroundings [1,2]. A classical example of such localized structures was first predicted by Turing [3]. Contrary to the Rayleigh-Bénard instability in fluids, this instability is characterized by an intrinsic wavelength, which depends only on the dynamical parameters of the considered system, such as diffusion coefficients and/or the inverse characteristic time associated with chemical kinetics. The theoretical support of the thermodynamics theory of systems far from equilibrium, developed by Prigogine, made the work of Turing popular. For this reason, it is worth renaming such a bifurcation a Turing-Prigogine instability. More than several decades later, de Kepper et al. [4] and Ouyang and Swinney [5] demonstrated experimental evidence of two-dimensional dissipative structures in 
chlorite-iodide-malonic acid reactions. It is also worth mentioning an earlier experimental work by Belousov, who was the first to observe the oscillatory behavior of chemical concentrations [6,7].

Dissipative structures are not necessarily periodic. They can be either isolated, randomly distributed or self-organized in clusters [8-16]. This spatial confinement comes from the balance between a positive feedback mechanism associated with the chemical reaction and a diffusion process. In addition, dissipation plays a stabilizing role when the system operates far from equilibrium (see recent overviews of this issue [17-20]). However, the circular shape of a single localized structure may exhibit a deformation followed by a fragmentation into two or more spots. This splitting process continues until the system reaches a hexagonal pattern. This phenomenon is referred to as spot multiplication, or self-replication [21]. Soon after this theoretical prediction, the spot replication phenomenon was experimentally observed in various experiments, such as the ferrocyanide-iodate-sulfite reaction [22], the Belousov-Zhabotinsky reaction [23-26] and the chloride dioxide-malonic acid reaction [27]. This phenomenon has been studied in different contexts of nonlinear science, such as biology [28], material science [29,30] and nonlinear optics [31]. It has been shown analytically that when the radius of the spot is small, the instability leads to a spot deformation, which generates only two spots [26]. See also [32-34] for the approach based on the interface motion.

In another line of research, the study of the formation of extreme events, such as rogue waves in nonlinear systems, is motivated by the experimental evidence of this phenomenon in fiber optics [35]. Several important papers and reviews of this active field of research have been published in recent years [36-54]. Rogue waves are rare events and giant pulses. Often, the long tail probability distribution is the fundamental characteristic accounting for the generation of rogue waves. They correspond to large intensity pulses in the spatial directions. Various mechanisms have been proven to be responsible for the generation of rogue waves in one-dimensional spatially-extended systems. In this paper, we propose a mechanism based on the delayed feedback control in reaction diffusion systems.

In this contribution, we show that in the absence of a delay feedback, the Brusselator model admits stationary localized structures in a finite range of parameters. When increasing the parameter $B$, localized structures become unstable with respect to curvature instability followed by an annulus structure. With the temporal evolution, the ring structure becomes unstable, leading to the formation of four spots. This process continues until the system reaches a hexagonal structure. When the delayed feedback is taken into account, we show that extreme events, often called rogue waves, may be generated in reaction-diffusion systems. The delayed feedback is found to induce a spontaneous formation of rogue waves. In the absence of the delay feedback, spatial spots are stationary. The rogue waves are excited and controlled by the delayed feedback. We characterize their formation by computing the probability distribution of the spot height. Indeed, the long-tailed statistical distribution, which is often considered as a signature of the presence of rogue waves, appears for sufficiently strong feedback.

The paper is organized as follows: in Section 2, we introduce the classical Brusselator model, and we show numerically the formation of localized structures, as well as the curvature instability leading to splitting. In Section 3, we introduce a retroaction loop in the Brusselator model and perform the linear stability analysis. We also analyze the formation of extreme events induced by the delayed feedback. We conclude in Section 4.

\section{Reaction-Diffusion Model and Spot Replication}

We consider the Brusselator [55] model as a classical paradigm of the theory of dissipative structures [1]. The evolution equations of the Brusselator model read:

$$
\begin{aligned}
& \frac{d X}{d t}=\nabla^{2} X+A-(B+1) X+X^{2} Y \\
& \frac{d Y}{d t}=D \nabla^{2} Y+B X-X^{2} Y .
\end{aligned}
$$


Here, $X$ and $Y$ denote the concentrations of the interacting chemical species. $A$ and $B$ are externally-controlled concentrations. The parameter $D$ is the ratio between the diffusion coefficients $D_{x}$ and $D_{y}$ of the $X$ and $Y$ concentrations, respectively. The homogeneous steady state $\left(X_{s}=A\right.$, $Y_{S}=B / A$ ) may become unstable with respect to a Turing-Prigogine instability that leads to periodic oscillations in the $(x, y)$ plane. A necessary condition for the Turing-Prigogine instability to occur is that the diffusion coefficient of the inhibitor $X$ species should be larger than that of the activator $Y$. The threshold and the critical wavenumber at the pattern forming instability are $B_{c}=(1+d A)^{2}$ and $k_{c}=\sqrt{d A}$, with $d=\sqrt{1 / D}=\sqrt{D_{x} / D_{y}}$ [1]. In two dimensions, a weakly-nonlinear analysis in the vicinity of the threshold associated with a pattern forming instability shows that when increasing the parameter $B$, the following sequence of patterns with different symmetry is generated: $H \pi$ (honeycomb lattice)-stripes- $H 0$ (triangular lattice) [56].
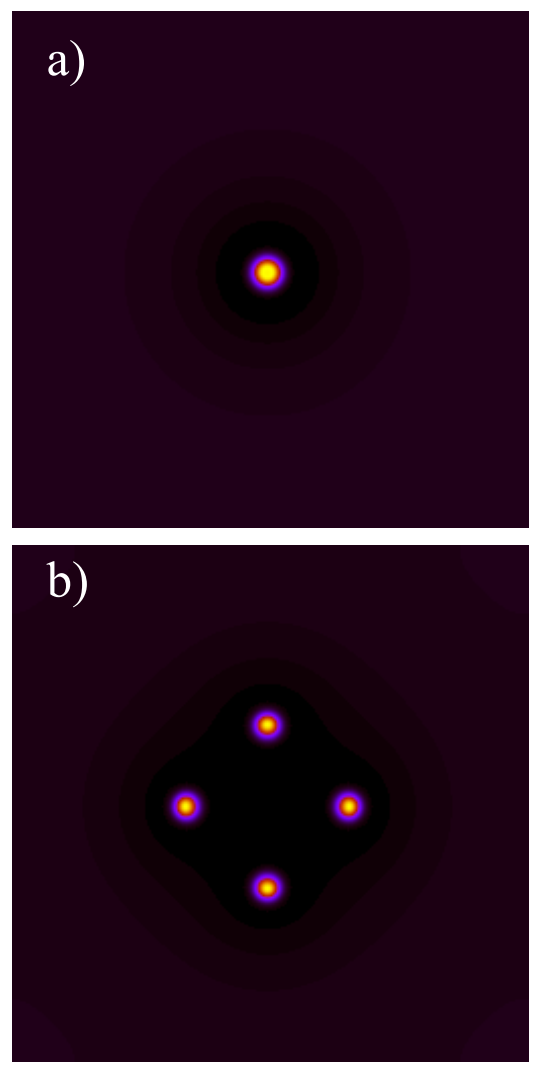

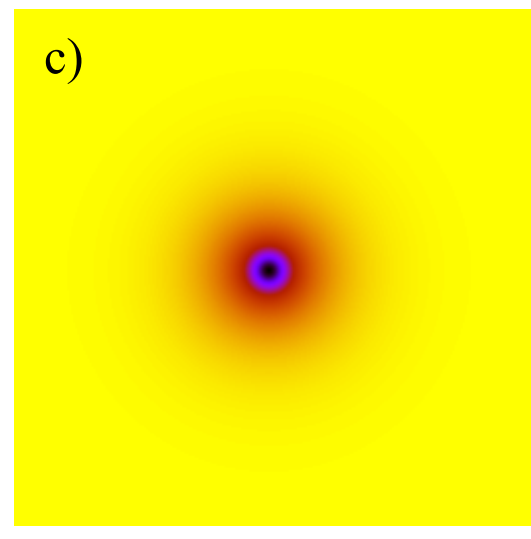

d)

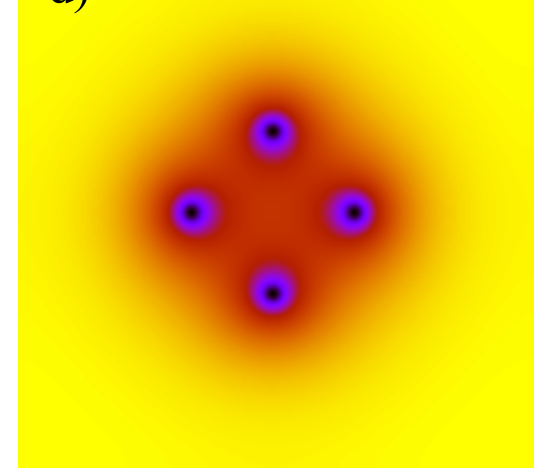

Figure 1. Examples of 2D stationary localized structures obtained from numerical simulations of the model Equation 1. (a) and (c) correspond to a single spot in the spatial profile of the chemical concentrations $X$ and $Y$, respectively. (b) and (d) correspond to four spots in the spatial profile of the chemical concentrations $X$ and $Y$, respectively. Parameters are $A=0.6, B=0.65$, and $D=150$. The mesh integration is $256 \times 256$ points.

Localized structures do not emerge spontaneously from the homogeneous steady states. They are generated with hard excitation (large amplitude initial perturbation) in the regime of coexistence between the homogeneous steady states and a periodic pattern. In this regime, the Brusselator model admits stationary localized structures. The initial condition solely determines the number and spatial location of spots. To generate a single spot, a Gaussian initial condition is used. An example of stationary localized spots with one and four peaks is shown in Figure 1. When increasing the parameter $B$, circular localized structures exhibit a self-replication phenomenon in a wide range of parameters. An example of this behavior is shown in Figure 2. During time evolution, a single localized spot becomes unstable and leads to the formation of an annulus (see Figure $2 \mathrm{~b}$ ). The annulus becomes unstable and breaks into four spots, as shown in Figure 2c. During time evolution, the four spots start 
to repel each other. The size of the new spots grows until it reaches a maximum size over which they exhibit an elliptical deformation followed by splitting into two daughter spots. This secondary splitting is shown in Figure 2e,f. Numerical simulations are obtained with periodic boundary conditions in both spatial dimensions.
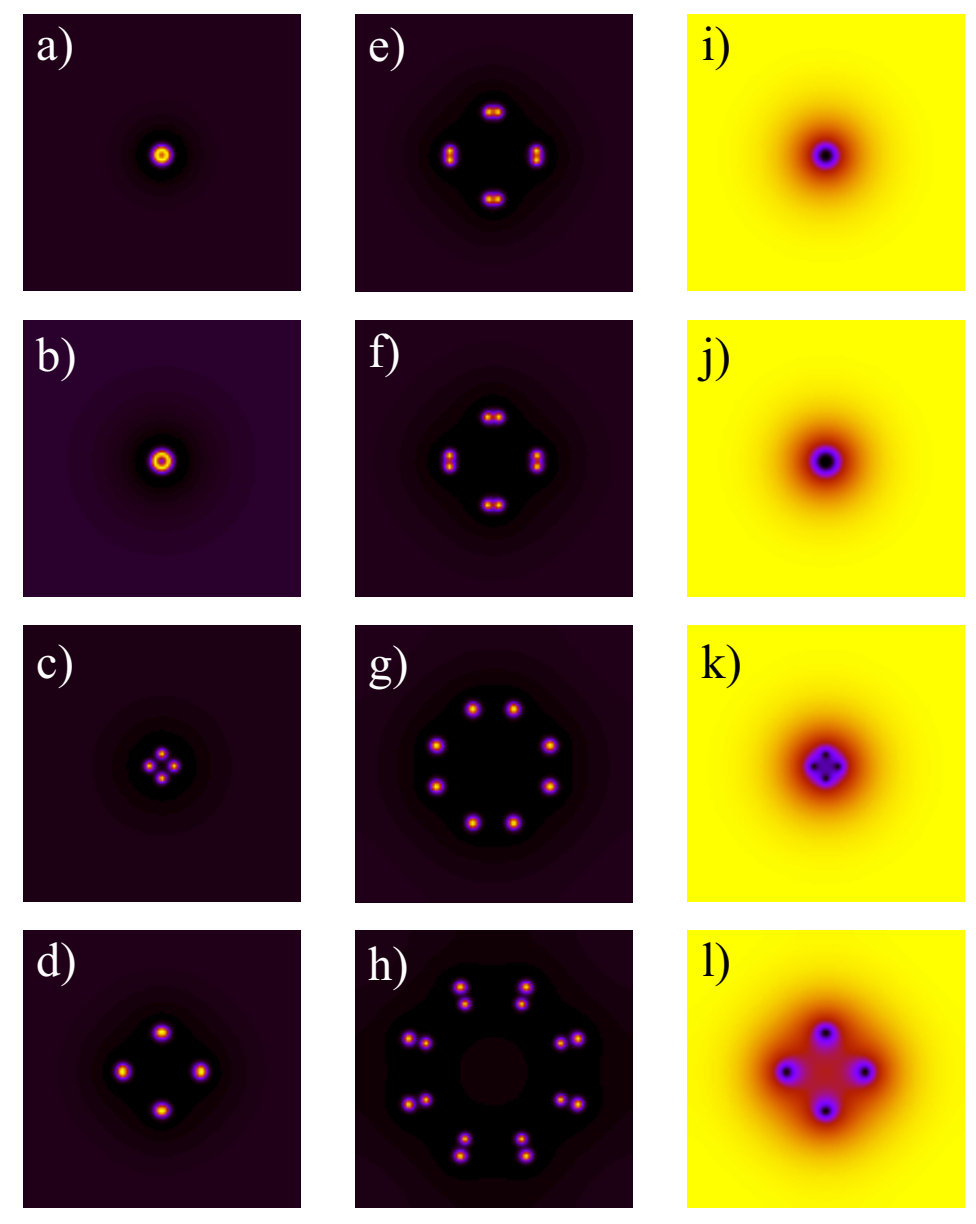

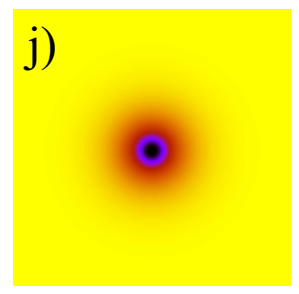

k)

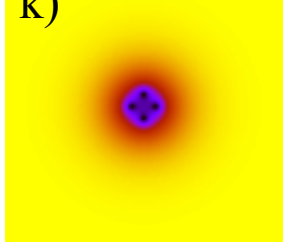

1)

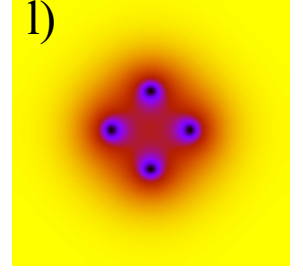

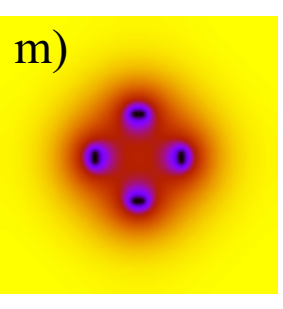

n)
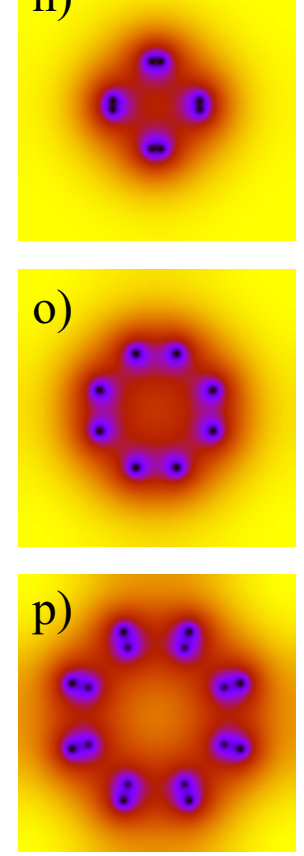

Figure 2. Self-replicating spots obtained from numerical simulations of the model Equation 1. (a)-(h): time evolution of the chemical concentration $X . \quad(\mathbf{i})-(\mathbf{p})$ : time evolution of the chemical concentration $Y$. Parameters are $A=0.6, B=0.75$, and $D=150$. The mesh integration is $256 \times 256$ points.

Note that the spots described by the variational Swift-Hohenberg equation can exhibit an elliptical deformation, leading to a rod-shaped structure. As it elongates, this structure exhibits a transversal instability that generates an invaginated labyrinth structure invading all of the space available [57].

\section{Extreme Events Induced by Delayed Feedback}

We incorporate in the Brusselator model Equation (1) a delay time effect, and we focus on a one-dimensional system. Recently, time-delayed feedback control in reaction-diffusion systems has attracted much interest [58-60]. The evolution equations of the Brusselator model with time-delayed feedback read:

$$
\begin{aligned}
& \frac{d X}{d t}=\frac{\partial X^{2}}{\partial x^{2}}+A-(B+1) X+X^{2} Y+\eta_{x} X(t-\tau) \\
& \frac{d Y}{d t}=D \frac{\partial Y^{2}}{\partial x^{2}}+B X-X^{2} Y+\eta_{y} Y(t-\tau) .
\end{aligned}
$$


The parameters $(A, B$ and $D)$ are defined in the previous section. The delayed feedback parameters are the strength of the delay $\left(\eta_{x}, \eta_{y}\right)$ and the delay time $\tau$. The approximation we use to model the delay is valid in the limit where the strength of the delay is small and the delay time is large. In this limit, the feedback concentrations are small enough for their evolution to be described by a single spatially-homogeneous delay term. In order to simplify the analysis, without loss of generality, we consider that $\eta_{x}=\eta$ and $\eta_{y}=0$. In this limit, the homogeneous steady states of the Brusselator model with delayed feedback read:

$$
X_{s}=\frac{A}{1-\eta}, \quad Y_{s}=\frac{B(1-\eta)}{A},
$$

The linear stability analysis with respect to a finite wavelength perturbation of the form $\exp (i k \cdot r+\lambda t)$ shows that the eigenvalue $\lambda$ vanishes when:

$$
D k^{4}+\left[(1-\eta-B) D+\frac{A^{2}}{(1-\eta)^{2}}\right] k^{2}+\frac{A^{2}}{1-\eta}=0 .
$$

Hence, there exists a finite band of Fourier modes $k$ with $k_{-}^{2}<k^{2}<k_{+}^{2}$, where $k_{-,+}^{2}$ are solutions of Equation (4). The threshold associated with the Turing-Prigogine instability, i.e., $k_{-} \stackrel{,}{=} k_{+}$, provides the threshold and the critical wavenumber at the onset of the instability:

$$
B_{c}=\frac{(1-\eta+d A)^{2}}{(1-\eta)}, \quad k_{c}^{2}=\frac{A \sqrt{1-\eta}}{1-\eta} d,
$$

with $d=\sqrt{1 / D}=\sqrt{D_{x} / D_{y}}$. The corresponding wavelength at the onset of the bifurcation point is:

$$
\Lambda_{c}=2 \pi\left(\frac{1-\eta}{d^{2} A^{2}}\right)^{1 / 4}
$$

In the absence of the delayed feedback, i.e., $\eta=0$, we recover the classical expressions for the threshold and the critical wavenumber at the pattern forming instability $B_{c}=(1+d A)^{2}$ and $k_{c}=\sqrt{d A}$ [1]. From the expressions of $B_{c}$ and the wavelength $\Lambda_{c}$, we see that both the threshold and the wavelength associated with the Turing-Prigogine instability are strongly affected by the delayed feedback strength. Figure 3 shows the critical value $B_{c}$ against the strength of the delayed feedback. For the fixed ratio between the diffusion coefficients, the threshold associated with pattern forming instability decreases with the increase of $\eta$ (excluding the region very close to $\eta=1$ ). The critical wavelength also decreases with respect to $\eta$ (see Figure 4).

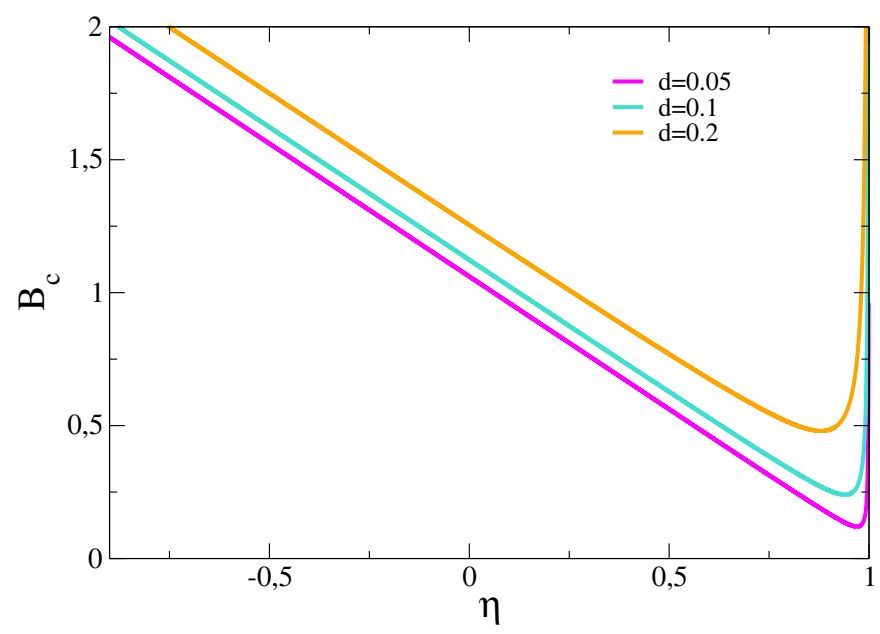

Figure 3. The threshold associated with the pattern formation instability as a function of the strength of the delayed feedback, with $A=0.6$. 


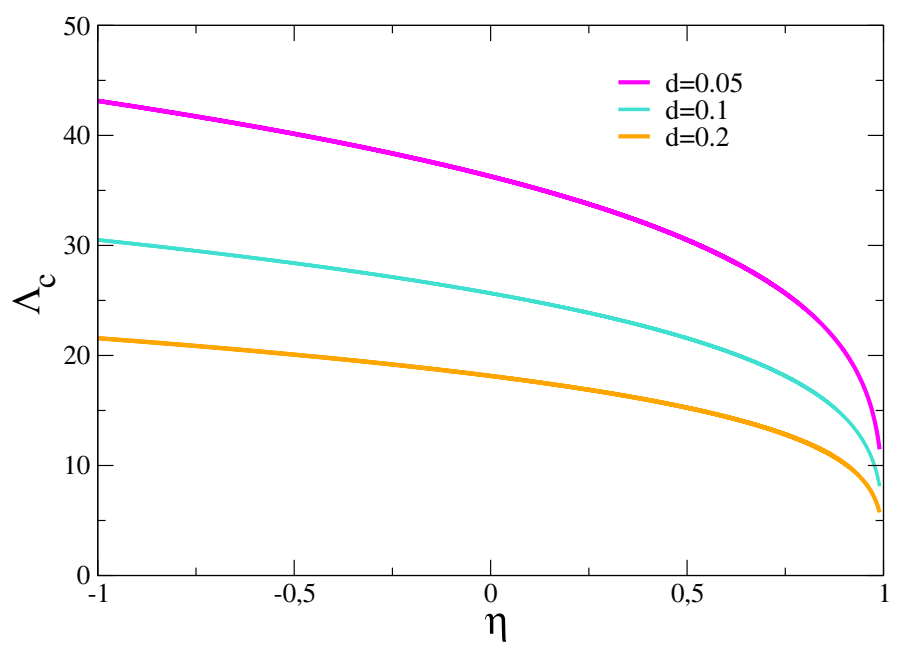

Figure 4. The wavelength of the Turing-Prigogine instability as a function of the strength of the delayed feedback, with $A=0.6$.

We now consider the effect of a delayed feedback in the one-dimensional Brusselator model. The delayed feedback allows for the motion of a stationary localized structure when the product of the delay time and the strength of the delay reach the numerical value $\eta \tau=1$ [58-61]. The phase of the delayed feedback may alter the threshold associated with the moving localized spots $[62,63]$. Delayed feedback could also induce the formation of localized chaos through the period doubling mechanism [64].

In this section, we show a novel way to generate and to control extreme events in reaction-diffusion systems by means of delayed feedback. Most of the papers published on rogue waves, either in the ocean or in optics, describe them in the framework of the nonlinear Schroedinger equation. However, we chose a system that cannot be described by the nonlinear Schroedinger equation and in which dissipation plays an important role. Furthermore, because the nonlinear Schroedinger equation does not admit localized two-dimensional solutions due to the collapse dynamics, most of the work has been limited to the one-dimensional setting. We establish that, while in the absence of delayed feedback, the spatial spots are stationary or exhibit a splitting and deformation, for sufficiently strong feedback, the spontaneous formation of extreme events is observed. These rogue waves are clearly excited and controlled by the feedback. We provide a statistical analysis showing a non-Gaussian profile of the probability distribution with a long tail and pulse intensity height well beyond two-times the significant wave height (SWH) defined as the average of one third of the highest amplitude pulses.

Numerical simulations with the periodic boundary condition of the Brusselator model with delayed feedback control (Equation (2)) are performed. A space-time map showing the evolution of $X(x, t)$ in the Brusselator model is presented in Figure $5 \mathrm{a}$, and a snapshot of the spatial distribution at $t=78$ capturing an extreme event is shown in Figure $5 \mathrm{~b}$. The Brusselator parameters are $D_{x}=1$, $D_{y}=150, A=0.6, B=0.75$, and the feedback parameters are $\eta_{x}=-\eta_{y}=2.5$ and $\tau=10$. As is evident from Figure 5a, pulses with intensity 5-10-times larger than that of the stationary localized structures without delayed feedback appear (indicated by triangles). An extreme event with amplitude $X>20$ is shown with a red square in Figure 5a. The cross-section of the space-time map showing the spatial profile of the rogue wave is shown in Figure $5 \mathrm{~b}$. In the absence of delayed feedback, the localized structure is a stationary object, as shown by a red line in Figure $5 b$. 

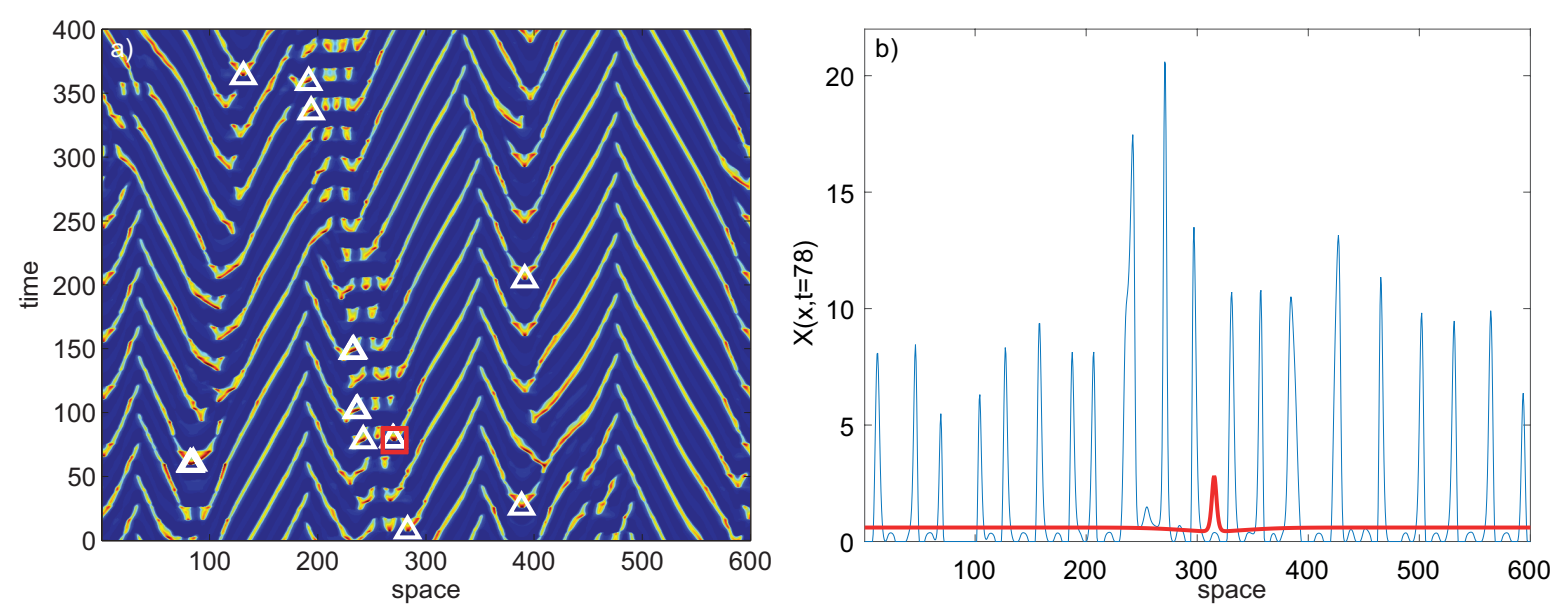

Figure 5. (a) Space-time map showing the evolution of $X(x, t)$ in the Brusselator reaction-diffusion model. Triangles indicate pulses with an intensity 5-10-times larger than the stationary localized structures without delayed feedback. The red square shows an extreme event with amplitude $X>20$. The Brusselator parameters are $D=150, A=0.6, B=0.75$, and the feedback parameters are $\eta_{x}=-\eta_{y}=2.5$ and $\tau=10$. (b) A cross-section at $\mathrm{t}=78$ showing the spatial profile $X(x)$ of the rogue wave.

Statistical analysis of the pulse height distribution of spatio-temporal chaos in the Brusselator model is presented in Figure 6. Here, SWH denotes the significant wave height defined as the average of one third of the highest amplitude pulses. The long-tailed statistical distribution serves as a signature of the presence of rogue waves: rogue waves with pulse heights more than twice the SWH appear in the system.

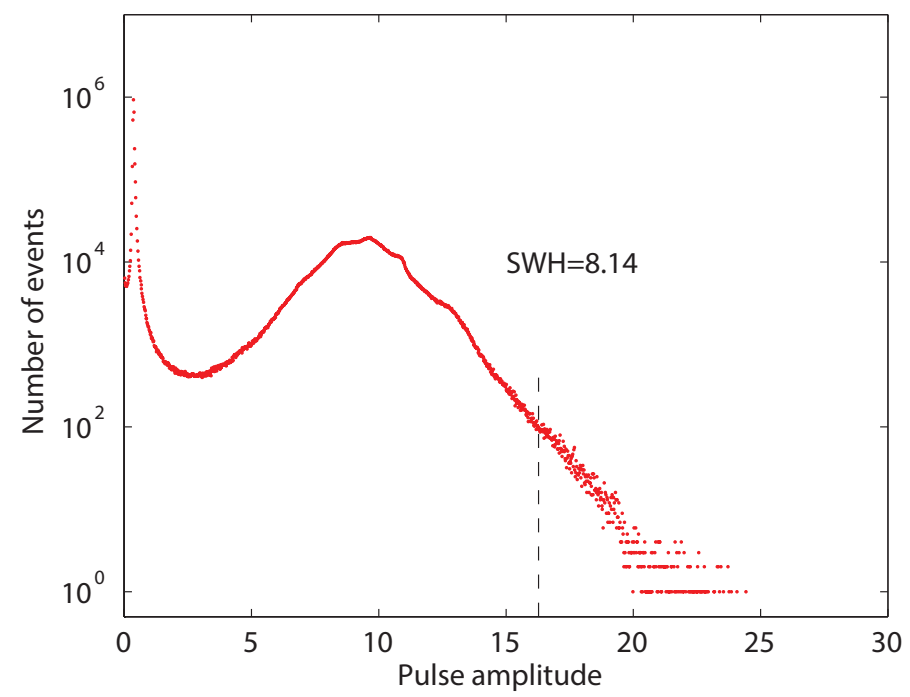

Figure 6. The number of events as a function of the amplitude of the pulses in the semi-logarithmic scale. The parameters are the same as in Figure 5. The SWH denotes the significant wave height. The dashed line indicates $2 \times \mathrm{SWH}$.

\section{Conclusions}

In the first part of this paper, we have shown numerically that the Brusselator model admits stationary localized spots. When the parameter $B$ is increased, the localized spots exhibit a self-replication phenomenon, leading to the formation of stationary hexagonal structures. In the second part, we have incorporated a time-delayed feedback loop in the Brusselator model in one 
dimension. We have shown that the time-delayed feedback leads to the formation of extreme events. This phenomenon occurs for large values of delayed feedback strength. We have characterized the formation of extreme events by providing a statistical analysis showing a non-Gaussian profile of the probability distribution with a long tail and spot intensity height well beyond two-times the significant wave height $(\mathrm{SWH})$ defined as the average of one third of the highest amplitude pulses. The generality of our analysis suggests that the instability leading to the spontaneous formation of rogue waves in a controllable way is a universal phenomenon. In future work, we plan to investigate the formation of extreme events in a two-dimensional Brusselator model with delayed feedback.

Acknowledgments: Mustapha Tlidi received support from the Fonds National de la Recherche Scientifique (Belgium), and Krassimir Panajotov is grateful to the Methusalem foundation for financial support.

Author Contributions: Mustapha Tlidi, Etienne Averlant and Krassimir Panajotov conducted the numerical investigations; Yerali Gandica performed the linear stability analysis. All authors participated in the writing and revising of the manuscript. All authors have read and approved the final manuscript.

Conflicts of Interest: The authors declare no conflict of interest.

\section{References}

1. Prigogine, I.; Lefever, R. Symmetry breaking instabilities in dissipative systems. II. J. Chem. Phys. 1968, 48, 1695-1700.

2. Glansdorff, P.; Prigogine, I. Thermodynamic Theory of Structure, Stability and Fluctuations; Wiley: Hoboken, NJ, USA, 1971.

3. Turing, A.M. The chemical basis of morphogenesis. Philos. Trans. R. Soc. Lond. B 1952, 237, 37-72.

4. Castets, V.; Dulos, E.; Boissonade, J.; de Kepper, P. Experimental evidence of a sustained standing Turing-type nonequilibrium chemical pattern. Phys. Rev. Lett. 1990, 64, 2953-2956.

5. Ouyang, Q.; Swinney, H.L. Transition from a uniform state to hexagonal and striped Turing patterns. Nature 1991, 352, 610-612.

6. Belousov, B.P. A periodic reaction and its mechanism. Compil. Abstr. Radiat. Med. 1959, 147, 1.

7. Zaikin, A.N.; Zhabotinsky, A.M. Concentration wave propagation in two-dimensional liquid-phase self-oscillating system. Nature 1970, 225, 535-537.

8. Koga, S.; Kuramoto, Y. Localized patterns in reaction-diffusion systems. Prog. Theor. Phys. 1980, 63, 106-121,

9. Pomeau, Y. Front motion, metastability and subcritical bifurcations in hydrodynamics. Phys. D 1986, 23, 3-11.

10. Rosanov, N.N.; Khodova, G.V. Autosolitons in bistable interferometers. Opt. Spectrosc. 1988, 65, 449-450.

11. Thual, O.; Fauve, S. Localized structures generated by subcritical instabilities. J. Phys. Fr. 1988, 49, $1829-1833$.

12. Malomed, B.A.; Nepomnyashchy, A.A. Kinks and solitons in the generalized Ginzburg-Landau equation. Phys. Rev. A 1990, 42, 6009-6014.

13. Tlidi, M.; Mandel, P.; Lefever, R. Localized structures and localized patterns in optical bistability. Phys. Rev. Lett. 1994, 73, 640-643.

14. Scroggie, A.J.; Firth, W.J.; McDonald, G.S.; Tlidi, M.; Lefever, R.; Lugiato, L.A. Pattern formation in a passive Kerr cavity. Chaos Solitons Fractals 1994, 4, 1323-1354.

15. Tlidi, M.; Lefever, R.; Vladimirov, A. On Vegetation Clustering, Localized Bare Soil Spots and Fairy Circles. In Dissipative Solitons: From Optics to Biology and Medicine; Springer: Berlin/Heidelberg, Germany, 2008; p. 751.

16. Vladimirov, A.G.; Lefever, R.; Tlidi, M. Relative stability of multipeak localized patterns of cavity solitons. Phys. Rev. A 2011, 84, 043848.

17. Dissipative Solitons: from Optics to Biology and Medicine; Akhmediev, N., Ankiewicz, A., Eds.; Springer: Berlin/Heidelberg, Germany, 2008; Volume 751.

18. Leblond, H.; Mihalache, D. Models of few optical cycle solitons beyond the slowly varying envelope approximation. Phys. Rep. 2013, 523, 61-126.

19. Tlidi, M.; Staliunas, K.; Panajotov, K.; Vladimirov, A.G.; Clerc, M.G. Localized structures in dissipative media: from optics to plant ecology. Philos. Trans. R. Soc. Lond. A 2014, 372, 20140101. 
20. Lugiato, L.; Prati, F.; Brambilla, M. Nonlinear Optical Systems; Cambridge University Press: Cambridge, UK, 2015.

21. Pearson, J.E. Complex patterns in a simple system. Science 1993, 261, 189-192.

22. Lee, K.J.; McCormick, W.D.; Pearson, J.E.; Swinney, H.L. Experimental observation of self-replicating spots in a reaction-diffusion system. Nature 1994, 369, 215-218.

23. Muñuzuri, A.P.; Pérez-Villar, V.; Markus, M. Splitting of Autowaves in an Active Medium. Phys. Rev. Lett. 1997, 79, 1941-1944.

24. Kaminaga, A.; Vanag, V.K.; Epstein, I.R. A reaction-diffusion memory device. Angew. Chem. Int. Ed. 2006, 45, 3087-3089.

25. Kaminaga, A.; Vanag, V.K.; Epstein, I.R. "Black spots" in a surfactant-rich Belousov-Zhabotinsky reaction dispersed in a water-in-oil microemulsion system. J. Chem. Phys. 2005, 122, 174706.

26. Kolokolnikov, T.; Tlidi, M. Spot deformation and replication in the two-dimensional Belousov-Zhabotinski reaction in a water-in-oil microemulsion. Phys. Rev. Lett. 2007, 98, 188303.

27. Davies, P.W.; Blanchedeau, P.; Dulos, E.; Kepper, P.D. Dividing Blobs, Chemical Flowers, and Patterned Islands in a Reaction-Diffusion System. J. Phys. Chem. A 1998, 102, 8236-8244,

28. Meron, E.; Gilad, E.; von Hardenberg, J.; Shachak, M.; Zarmi, Y. Vegetation patterns along a rainfall gradient. Chaos Solitons Fractals 2004, 19, 367-376.

29. Ren, X.; Wei, J. On the spectra of three-dimensional lamellar solutions of the diblock copolymer problem. SIAM J. Math. Anal. 2003, 35, 1-32, doi:10.1137/S0036141002413348.

30. Nishiura, Y.; Suzuki, H. Higher Dimensional SLEP Equation and Applications to Morphological Stability in Polymer Problems. SIAM J. Math. Anal. 2005, 36, 916-966,

31. Tlidi, M.; Vladimirov, A.G.; Mandel, P. Curvature Instability in Passive Diffractive Resonators. Phys. Rev. Lett. 2002, 89, 233901.

32. Muratov, C.B.; Osipov, V.V. General theory of instabilities for patterns with sharp interfaces in reaction-diffusion systems. Phys. Rev. E 1996, 53, 3101-3116.

33. Hayase, Y.; Ohta, T. Sierpinski Gasket in a Reaction-Diffusion System. Phys. Rev. Lett. 1998, 81, $1726-1729$.

34. Hayase, Y.; Ohta, T. Self-replicating pulses and Sierpinski gaskets in excitable media. Phys. Rev. E 2000, 62, 5998-6003.

35. Solli, D.R.; Ropers, C.; Koonath, P.; Jalali, B. Optical rogue waves. Nature 2007, 450, 1054-1057.

36. Kibler, B.; Fatome, J.; Finot, C. Millot, G.; Dias, F.; Genty, G.; Akhmediev, N.; Dudley, J.M. The Peregrine soliton in nonlinear fibre optics. Nat. Phys. 2010, 6, 790-795.

37. Chabchoub, A.; Hoffmann, N.P.; Akhmediev, N. Rogue Wave Observation in a Water Wave Tank. Phys. Rev. Lett. 2011, 106, 204502.

38. Akhmediev, N.; Soto-Crespo, J.; Ankiewicz, A. Extreme waves that appear from nowhere: On the nature of rogue waves. Phys. Lett. A 2009, 373, 2137-2145.

39. Akhmediev, N.; Dudley, J.M.; Solli, D.R.; Turitsyn, S.K. Recent progress in investigating optical rogue waves. J. Opt. 2013, 15, 060201.

40. Onorato, M.; Residori, S.; Bortolozzo, U.; Montina, A.; Arecchi, F. Rogue waves and their generating mechanisms in different physical contexts. Phys. Rep. 2013, 528, 47-89.

41. Dudley, J.M.; Dias, F.; Erkintalo, M.; Genty, G. Instabilities, breathers and rogue waves in optics. Nat. Photon. 2014, 8, 755-764.

42. Mussot, A.; Louvergneaux, E.; Akhmediev, N.; Reynaud, F.; Delage, L.; Taki, M. Optical Fiber Systems Are Convectively Unstable. Phys. Rev. Lett. 2008, 101, 113904.

43. Odent, V.; Taki, M.; Louvergneaux, E. Experimental spatial rogue patterns in an optical feedback system. Nat. Hazards Earth Syst. Sci. 2010, 10, 2727-2732.

44. Arecchi, F.T.; Bortolozzo, U.; Montina, A.; Residori, S. Granularity and Inhomogeneity Are the Joint Generators of Optical Rogue Waves. Phys. Rev. Lett. 2011, 106, 153901.

45. Birkholz, S.; Nibbering, E.T.J.; Brée, C.; Skupin, S.; Demircan, A.; Genty, G.; Steinmeyer, G. Spatiotemporal Rogue Events in Optical Multiple Filamentation. Phys. Rev. Lett. 2013, 111, 243903.

46. Montina, A.; Bortolozzo, U.; Residori, S.; Arecchi, F.T. Non-Gaussian Statistics and Extreme Waves in a Nonlinear Optical Cavity. Phys. Rev. Lett. 2009, 103, 173901.

47. Lushnikov, P.M.; Vladimirova, N. Non-Gaussian statistics of multiple filamentation. Opt. Lett. 2010, 35 , 1965-1967. 
48. Marsal, N.; Caullet, V.; Wolfersberger, D.; Sciamanna, M. Spatial rogue waves in a photorefractive pattern-forming system. Opt. Lett. 2014, 39, 3690-3693.

49. Baronio, F.; Conforti, M.; Degasperis, A.; Lombardo, S.; Onorato, M.; Wabnitz, S. Vector Rogue Waves and Baseband Modulation Instability in the Defocusing Regime. Phys. Rev. Lett. 2014, 113, 034101.

50. Leonetti, M.; Conti, C. Observation of three dimensional optical rogue waves through obstacles. Appl. Phys. Lett. 2015, 106, 254103.

51. Pierangeli, D.; di Mei, F.; Conti, C.; Agranat, A.J.; DelRe, E. Spatial Rogue Waves in Photorefractive Ferroelectrics. Phys. Rev. Lett. 2015, 115, 093901.

52. Zhong, W.P.; Belić, M.; Zhang, Y. Second-order rogue wave breathers in the nonlinear Schrödinger equation with quadratic potential modulated by a spatially-varying diffraction coefficient. Opt. Express 2015, 23, 3708-3716.

53. Bonatto, C.; Feyereisen, M.; Barland, S.; Giudici, M.; Masoller, C.; Leite, J.R.R.; Tredicce, J.R. Deterministic Optical Rogue Waves. Phys. Rev. Lett. 2011, 107, 053901.

54. Reinoso, J.A.; Zamora-Munt, J.; Masoller, C. Extreme intensity pulses in a semiconductor laser with a short external cavity. Phys. Rev. E 2013, 87, 062913.

55. Tyson, J.J. Some further studies of nonlinear oscillations in chemical systems. J. Chem. Phys. 1973, 58, 3919-3930.

56. Verdasca, J.; de Wit, A.; Dewel, G.; Borckmans, P. Reentrant hexagonal Turing structures. Phys. Lett. A 1992, 168, 194-198.

57. Bordeu, I.; Clerc, M.G.; Lefever, R.; Tlidi, M. From localized spots to the formation of invaginated labyrinthine structures in a Swift-Hohenberg model. Commun. Nonlinear Sci. Numer. Simul. 2015, 29, 482-487.

58. Tlidi, M.; Sonnino, A.; Sonnino, G. Delayed feedback induces motion of localized spots in reaction-diffusion systems. Phys. Rev. E 2013, 87, 042918.

59. Gurevich, S.V.; Friedrich, R. Instabilities of Localized Structures in Dissipative Systems with Delayed Feedback. Phys. Rev. Lett. 2013, 110, 014101.

60. Gurevich, S.V. Dynamics of localized structures in reaction-diffusion systems induced by delayed feedback. Phys. Rev. E 2013, 87, 052922.

61. Tlidi, M.; Vladimirov, A.G.; Pieroux, D.; Turaev, D. Spontaneous Motion of Cavity Solitons Induced by a Delayed Feedback. Phys. Rev. Lett. 2009, 103, 103904.

62. Panajotov, K.; Tlidi, M. Spontaneous motion of cavity solitons in vertical-cavity lasers subject to optical injection and to delayed feedback. Eur. Phys. J. D 2010, 59, 67-72.

63. Pimenov, A.; Vladimirov, A.G.; Gurevich, S.V.; Panajotov, K.; Huyet, G.; Tlidi, M. Delayed feedback control of self-mobile cavity solitons. Phys. Rev. A 2013, 88, 053830.

64. Panajotov, K.; Tlidi, M. Chaotic behavior of cavity solitons induced by time delay feedback. Opt. Lett. 2014, $39,4739-4742$.

(C) 2016 by the authors; licensee MDPI, Basel, Switzerland. This article is an open access article distributed under the terms and conditions of the Creative Commons by Attribution (CC-BY) license (http:/ / creativecommons.org/licenses/by/4.0/). 